\title{
The Ethics of Coexistence: \\ Can I Learn to Stop Worrying and Love the Logic Bomb?
}

\author{
John Aycock \\ Department of Computer Science, University of Calgary, \\ 2500 University Drive NW, Calgary, Alberta, Canada T2N 1N4 \\ aycock@ucalgary.ca \\ Anil Somayaji \\ School of Computer Science, Carleton University, \\ 1125 Colonel By Drive, Ottawa, Ontario, Canada K1S 5B6 \\ soma@scs.carleton.ca \\ John Sullins \\ Department of Philosophy, Sonoma State University, \\ 1801 East Cotati Ave., Rohnert Park, CA 94928 \\ john.sullins@sonoma.edu
}

Working paper: TR 2010-986-35, December 2010

\begin{abstract}
The ethics of computer security defenses are not often examined. We look at the ethics of defenses allowing malware to coexist within a single machine and within a broader community, gaining insight on the ethics of traditional defenses as a side effect.
\end{abstract}

\section{Introduction}

Ethical issues are much easier to examine and debate when there is obvious harm that may result from actions and consequences: euthanasia, slavery, weapons development. The same principle seems to hold in the ethics of computer security. Virus writing is potentially harmful, for example, and elicits ethical analyses - indeed, it appears impossible to write a text on computer ethics without a discussion about computer viruses [7, 11, 16, 28]. Whether "good" viruses and worms can exist has also been examined from the viewpoint of ethics [5, 9], as has proactive research looking to identify new security threats $[4,6]$. But there is no need to examine computer security 
defenses from an ethical point of view because they are always doing good... or are they?

As a simple example, a web site's passwords are too easy to guess, so an administrator concerned about brute-force and dictionary attacks installs software that requires users to make strong passwords. Users, in turn, respond by writing passwords down [1] and reusing passwords [15]; attackers switch to phishing and keylogging to steal passwords, attacks that can no longer be detected by the web site's server. This example involves choice, an action taken in the name of security, an action with consequences. As such, it may be subjected to ethical analysis, as can computer security defenses in general.

Most current defenses implicitly assume that the world is divided into two parts: the protected part and the unprotected part. Defense mechanisms attempt to keep the protected part from being compromised; at best, they have no effect on the unprotected part. Malware clearly coexists with legitimate software in current defense scenarios, but (ideally) in the unprotected part. In this paper we look at whether malware coexistence can be extended to apply within a single system and still retain security for the user, using a new type of system called a cosecure system. Cosecure systems pose a number of ethical challenges, which we use to give insight into traditional defenses and point towards alternative defense approaches.

The rest of this paper proceeds as follows. Section 2 introduces the concept of cosecure systems. We perform an ethical analysis of cosecure systems in Section 3; Section 4 looks at an alternative approach, and Section 5 concludes.

\section{Cosecure Systems}

Current approaches to defend computers against threats would make a great deal of sense to anyone from medieval times. Erect some walls (more walls are better) and place everything to be protected inside the walls. A breach of the walls is disaster.

Firewalls blocking inbound connections are a perfect embodiment of this metaphor, of course, as are Trojan horses being used to break through defenses. The other current defensive mainstay, anti-virus software, effectively builds walls too by scanning files, memory, and incoming packets to keep threats out. This idea of protection is reflected culturally, as Johnston observes [17, page 24]: 'The antivirus industry's grand narrative is that it is successfully producing antidotes that update computer software against global viral threats.'

How 'successfully' these defenses work is open to debate. A firewall provides no protection from users running malicious code behind the firewall via drive-by downloads and/or social engineering. Anti-virus software relies heavily on a reactive model ${ }^{1}$ that is increasingly lagging behind the onslaught of barbarian malware assailing the walls $[12,13,22]$. In any case, anti-virus software can never fully succeed because its task has long been known to be undecidable [10]. Even the notion of multiple walls defense-in-depth - is being called into question [21].

\footnotetext{
${ }^{1}$ We recognize that anti-virus heuristics, for example, may detect unknown malware proactively, but it is still fair to say that anti-virus is largely reactive. See [3, 27] for more on anti-virus software.
} 
These strategies share an all-or-nothing approach to defense, where the goal is to keep all computers malware-free. An alternative is to look at the larger population of computers, accept that some of those computers will be afflicted by malware, and work on containing the problem to those computers. Some work has been done on this (e.g., virus throttling [29]), but the approach is not widespread and it still conceptually treats a computer's status as binary: infected or not infected. ${ }^{2}$

We are proposing a different approach in this paper. We argue that it is increasingly unrealistic to assume that a computer can be kept free of malware, and we must build systems where legitimate software is able to coexist with malicious software. ${ }^{3}$ The challenge is to construct a system that permits a user to use the computer and have their data and actions uncompromised, yet also permits malware to be present and functional. We call such a computer not secure, not insecure, but cosecure. By way of analogy, consider a city. A city is well known to have crime taking place within it, and dark alleys better left unexplored, yet remains functioning regardless.

While there may be great technical challenges in building a cosecure system, we assume for purposes of ethical analysis that we are able to build one. What are the ethical implications? Furthermore, the details of this system have been left purposely vague; can the cosecure system design follow from ethical constraints?

\section{Ethics and the Cosecure System}

From a conceptual, information ethics standpoint we can argue for the use of a cosecure system. Information entities can all be said to have a certain amount of intrinsic worth. It is only on close analysis of the code and a complete explanation of the computing environment the code is functioning in that any sort of judgment of the value of the code can be reached. This task is difficult, if not impossible, so our system defaults to one that acknowledges that any code may have some worth. But we are also not claiming that every piece of code in a user's system is benign to that user. Some will be working against their wishes, some will be working for their interests, and some will have neutral value. Thus we can see that very complex mixtures of harm, help, and ambivalence can occur from the point of view of all the various applications in the system.

The idea of a cosecure system also shines a different light on security. Specifically, are security professionals (and their progeny, security software) ethically obligated to provide full defense at all times? Other professions are not, such as police or rescue personnel; they are obligated to do what they can but no one expects perfection. It seems that we place stronger demands on computer security, perhaps in part due to the differences between the physics of the real world (police can't be everywhere) versus the cyber world, in part due to a single security breach being potentially disastrous.

Certainly expectations on security software are high. Anti-virus software is used in computer forensics (e.g., [30]), for example, and as such its results need to be accurate enough to stand up in court. At the same time anti-virus and other security software

\footnotetext{
${ }^{2}$ Or, in keeping with the virus throttling work, misbehaving or behaving.

${ }^{3}$ Kursawe and Katzenbeisser [19] also considered coexistence, but did not examine ethical issues at all.
} 
are known to security professionals to yield an imperfect defense, as discussed in Section 2. A cosecure system is more ethical than traditional systems in the sense that its very concept is honest and forthcoming in terms of the security it provides and does not provide, thus upholding a duty to be truthful on the part of security professionals. ${ }^{4}$ While it can be argued that at certain times a half truth or "Platonic" lie might be justifiable, in this case there is no clear benefit to the user told the half truth that their system is fully protected by the purchase and installation of anti-virus software. There is no known placebo effect possible in this situation and in fact it works in the reverse. The user can operate their system with no idea about the presence of malware operating behind the scenes and beyond the defenses of anti-virus software. The only beneficiaries in these situations are the people selling the software and the people making the malware.

Another commonly-stated duty for computer professionals (e.g., [2, 14]), system administrators (e.g., [31]), and users alike (enshrined in the legal principle of duty of care) is avoiding harm to others and not endangering the public. We must then consider if a cosecure system would violate this duty. The answer derives from what the malware on a cosecure system is permitted to do: malware that attacks other computers by attempting remote exploits for worm spread, or joins a distributed denial-of-service attack clearly causes harm to others. Malware that does not overtly attack is a bit more subtle ethically, but - for example - malware that proxies connections to a "mother ship" for purposes of phishing infrastructure [24] is not ethically neutral in that it facilitates harm being done.

It follows that there must be constraints on the malware in a cosecure system in order for the system to operate in an ethical fashion. This substantially informs a cosecure system's design. A cosecure system must be able to enforce constraints and must therefore have some form of unimpeachable executive provided through a secure operating system kernel or virtualization. This may further extend to having a trusted, secure boot process.

For the remainder of the cosecure system design, we turn to the basic tenets of security: confidentiality, integrity, and availability [8,23]. As these underpin our notion of security, an ethical case can be made that a cosecure system has a duty to provide these to a legitimate user.

Availability of a cosecure system addresses whether or not a user has the appropriate resources (e.g., CPU time, memory, bandwidth) for their legitimate computer usage. Resource scheduling is well-studied in operating systems; looking at CPU time, for instance, a cosecure system could employ lottery scheduling [32] with the user possessing the majority of "tickets," which can then be passed as necessary to other processes acting on behalf of the user.

Preserving integrity in a cosecure system implies that a user's programs and data cannot be modified by the malware sharing the system. ${ }^{5}$ Access control plays an im-

\footnotetext{
${ }^{4}$ While not binding on security professionals, we note that this is a general moral imperative in the ACM Code of Ethics ('Be honest and trustworthy' [2]), and is also reflected in the IEEE Code of Ethics ('... be honest and realistic in stating claims ... based on available data' [14]).

${ }^{5}$ Data integrity and confidentiality could be addressed in part by assuming that a user's data is stored somewhere in the cloud, but then attacks involving the malware getting access to or modifying the cloud data would have to be prevented. We omit further discussion of this variant design due to space constraints.
} 
portant role here - changes are permitted only on the behest of the legitimate user and a secure software update process can be used to prevent malicious updates being introduced [33].

Access control also figures prominently in confidentiality in a cosecure system. A legitimate user's data cannot be allowed to be exfiltrated by malware in any way. This obviously includes local data, but also data dynamically generated: webcam images, microphone data, keystrokes, mouse clicks, screen shots, network traffic. In essence, only the user must be allowed access to cosecure system I/O. Even supposedly isolated malware processes cannot access user data, because of the risk of leaking it via a covert channel [20].

What does this leave? Any process not initiated by a legitimate user cannot access data on a system, except for its own, and has no I/O access apart from perhaps a limited amount of network bandwidth (limited to deter DDoS attacks and other nefarious uses). In essence, we are left with only a (distributed) computing platform for malware, eerily reminiscent of Shoch and Hupp's "worms" at Xerox PARC [25].

We have also ignored an important point, namely how malware gets onto a cosecure system to begin with. If the malware is present on the system initially, then it is insecure, not cosecure, at the outset. If a user explicitly installs the malware, perhaps through social engineering, then the malware is acting on behalf of the user and is legitimate, in the cosecure system sense. If the malware is injected into a legitimate user application, then again the malware will appear to be acting on behalf of a legitimate user. We therefore assert that, strictly following ethical design constraints, a cosecure system cannot be built.

A cosecure system is still useful, however, because it allows us to view traditional systems anew. There is no meaningful way for malware and legitimate users to coexist on the same machine even if the system is expressly designed to allow it, and have ethical duties upheld: duties to not harm others, and duties to the user to provide confidentiality, integrity, and availability. The cosecure system essentially acts as a proof by contradiction. In practice, of course, the situation is even worse - traditional systems are not as restricted as our mythical cosecure system, and systems with "secure" booting, "secure" kernels, and "secure" virtualization are invariably found to be subvertible (e.g., $[18,26])$.

Herein lies the dilemma. On one hand, we cannot observe ethical duties if malware is allowed on a user's computer; on the other hand, we know that current defenses are flawed. Nor are we absolved from this dilemma by defense in depth. Piling defenses - flawed or specialized to a single type of attack, or both - haphazardly together and trusting to blind faith that invaders will be kept at bay is not an ethically laudable position either.

One could arrive at the conclusion that the only solution is to do nothing defensively, but given the potential for direct harm to the user and indirect harm to others, this is not ethically permissible. We argue that there is an ethical obligation to apply current defenses even though, being imperfect, they cannot completely fulfill duties to the user. The obligation arises only because applying current defenses is more ethical than doing nothing. This is not unlike voting against bad candidates in an election rather than voting for a good candidate. It is a distasteful situation to be in, as a security professional, and we are forced to ask if there are alternatives. 


\section{An Alternative Approach}

To begin with, building walls and creating a fortress of a single machine (traditional defenses) or within a single machine (cosecure systems) is problematic from an information ethics point of view. Consider a physical building in a large city. Every building, even a private home, has some property that others can use, such as sidewalks and other areas where use is welcome, e.g., walking up to the door when the mail is delivered or if a friend visits, but is suspicious when strangers approach. But it is impossible, and even undesirable for a private home to attempt to become a fortress. It is antagonistic to the free flow of concord and commerce in the public sector. In the world of code, fortress mentality works to thwart the benefits of free and open computing and this limits the growth of valuable information entities.

If the single computer as fortress is undesirable and unachievable, then we must look more broadly to the single computer as an entity in a community which permits the free flow of information within it. We already organize our computers into communities by networking them, and we should be organizing our defenses to reflect this too. The key missing concept in security terms is that of the group: collective responsibility. Individual computers in a network should not just be responsible for their own security; they must also help keep other machines secure. Similarly, system administrators have a responsibility to not just keep their systems safe but to keep other members of the community - the Internet - safe when they can [31]. Current mechanisms, however, are more based on a "rugged individualism" view of the world where everybody takes care of their own but nobody has a responsibility to anybody else.

Space limitations preclude a full discussion and analysis of this alternative approach, but in practice, principles for community defense include the following:

- Information about attempted or successful security breaches must be disseminated widely in a timely fashion.

- Attacked systems must strive to prevent the attacker from targeting others.

- Systems should leave a forensic trail so others can learn from their demise.

- Systems must monitor their neighbors.

- Systems may disable themselves altruistically if doing so helps preserve the community.

Note that we are not assuming that all members of the community will be malwarefree. This releases us from ethical problems related to imperfect defenses for any given user, as our duty is now to the larger community, where we have no choice but to coexist with malware. The altered security perspective also allows us to draw on ethical arguments developed for actions and consequences within human communities.

\section{Conclusion}

We cannot stop worrying about malware, and coexistence with malware on a single machine is not ethically tenable. Looking to coexistence within a larger community is 
one solution, but tolerating and embracing the inevitability of malware is a strange love indeed.

\section{Acknowledgment}

The first two authors' research is supported in part by the Natural Sciences and Engineering Council of Canada via ISSNet, the Internetworked Systems Security Network.

\section{References}

[1] A. Adams and M. A. Sasse. Users are not the enemy. Communications of the ACM, 42(12):40-46, 1999.

[2] Association for Computing Machinery. ACM code of ethics and professional conduct. http://www.acm.org/about/code-of-ethics, 1992.

[3] J. Aycock. Computer Viruses and Malware. Springer, 2006.

[4] J. Aycock and A. Maurushat. Future threats. In 17th Virus Bulletin International Conference, pages 275-281, 2007.

[5] J. Aycock and A. Maurushat. "Good" worms and human rights. ACM SIGCAS Computers and Society, 38(1):28-39, 2008.

[6] J. Aycock and J. Sullins. Ethical proactive threat research. In Workshop on Ethics in Computer Security Research, pages 231-239, 2010.

[7] S. Baase. A Gift of Fire. Prentice Hall, 2nd edition, 2003.

[8] M. Bishop. Computer Security: Art and Science. Addison Wesley, 2003.

[9] V. Bontchev. Are "good" computer viruses still a bad idea? In Proceedings of the EICAR '94 Conference, pages 25-47, 1994.

[10] F. Cohen. Computer viruses: Theory and experiments. Computers $\mathcal{F}$ Security, 6(1):22-35, 1987.

[11] T. Forester and P. Morrison. Computer Ethics. MIT Press, 2nd edition, 1994.

[12] K. J. Higgins. Antivirus rarely catches Zbot Zeus Trojan. Dark Reading, 16 September 2009.

[13] K. J. Higgins. Study: Antivirus software catches about half of malware, misses 15 percent altogether. Dark Reading, 2 March 2009.

[14] Institute of Electrical and Electronics Engineers. IEEE code of ethics. http://www.ieee.org/portal/pages/iportals/aboutus/ethics/code.html, 2006.

[15] B. Ives, K. R. Walsh, and H. Schneider. The domino effect of password reuse. Communications of the ACM, 47(4):75-78, 2004. 
[16] D. G. Johnson. Computer Ethics. Prentice Hall, 3rd edition, 2001.

[17] J. Johnston. Technological Turf Wars: A Case Study of the Computer Antivirus Industry. Temple University Press, 2009.

[18] S. T. King, P. M. Chen, Y.-M. Wang, C. Verbowski, H. J. Wang, and J. R. Lorch. SubVirt: Implementing malware with virtual machines. In 2006 IEEE Symposium on Security and Privacy, pages 314-327, 2006.

[19] K. Kursawe and S. Katzenbeisser. Computing under occupation. In New Security Paradigms Workshop, pages 81-88, 2007.

[20] B. W. Lampson. A note on the confinement problem. Communications of the ACM, 16(10):613-615, 1973.

[21] M. E. Locasto, S. Bratus, and B. Schulte. Bickering in-depth: Rethinking the composition of competing security systems. IEEE Security $\mathcal{E}$ Privacy, pages $77-$ 81, November/December 2009.

[22] J. Oberheide, E. Cooke, and F. Jahanian. CloudAV: N-version antivirus in the network cloud. In 17th USENIX Security Symposium, pages 91-106, 2008.

[23] C. P. Pfleeger and S. L. Pfleeger. Security in Computing. Prentice Hall, 3rd edition, 2003.

[24] Honeynet Project. Know your enemy: Fast-flux service networks, 2007.

[25] J. F. Shoch and J. A. Hupp. The "worm" programs - early experience with a distributed computation. Communications of the ACM, 25(3):172-180, 1982.

[26] M. Steil. 17 mistakes Microsoft made in the Xbox security system. In 22nd Chaos Communication Congress, 2005.

[27] P. Szor. The Art of Computer Virus Research and Defense. Addison-Wesley, 2005.

[28] H. T. Tavani. Ethics $\mathcal{E}$ Technology: Ethical Issues in an Age of Information and Communication Technology. Wiley, 2004.

[29] J. Twycross and M. M. Williamson. Implementing and testing a virus throttle. In Proceedings of the 12th USENIX Security Symposium, pages 285-294, 2003.

[30] United States District Court, D. Puerto Rico. N. Rivera-Cruz v. Latimer, Biaggi, Rachid \& Godreau, LLP, et al. Civil No. 04-2377 (ADC), 16 June 2008.

[31] USENIX Special Interest Group for Sysadmins (SAGE). System administrators' code of ethics. http://www.sage.org/ethics, 2003.

[32] C. A. Waldspurger and W. E. Weihl. Lottery scheduling: Flexible proportionalshare resource management. In 1st Symposium on Operating Systems Design and Implementation, 1994. 
[33] G. Wurster and P. C. van Oorschot. Self-signed executables: Restricting replacement of program binaries by malware. In 2nd USENIX Workshop on Hot Topics in Security, 2007. 